\title{
REPRESENTATIVIDAD, DISPONIBILIDAD Y SOBRECONFIANZA: LAS HEURÍSTICAS DE LOS EMPRESARIOS PYME ${ }^{1}$
}

\author{
REPRESENTATIVITY, AVAILABILITY Y OVER-CONFIDENCE: \\ THE SME ENTREPRENEURS' HEURISTICS
}

\author{
Melisa Manzanal ${ }^{2}$, Gastón Milanesi ${ }^{3}$, Hernán Vigier ${ }^{4}$
}

\section{Resumen}

El presente trabajo forma parte de una investigación mayor que buscó caracterizar la toma de decisiones de los empresarios de pymes de Bahía Blanca, Argentina. En las últimas décadas, estudios mayormente experimentales han probado la existencia de heurísticas y sesgos en el accionar humano. Por consiguiente, el objetivo de este artículo fue determinar qué heurísticas son más propensas a presentarse en las elecciones de un universo particular de sujetos: los empresarios de pymes de la citada ciudad. En consecuencia, se recurrió a un panel de expertos en pymes. Para el procesamiento de los datos en él obtenidos se emplearon dos técnicas: probabilística (frecuencias absolutas y acumuladas) y no probabilística o borrosa (método directo con múltiples expertos). Se concluye que las

\footnotetext{
${ }^{1}$ Este artículo constituye un resumen de la primera parte de la tesis de Doctorado en Ciencias de la Administración (UNS) de Melisa Manzanal, dirigida por Gastón Milanesi y Hernán Vigier. La misma fue defendida y aprobada el 23/03/2017 (Expte. 2417/2012). El trabajo de tesis fue desarrollado en el marco de una beca doctoral del CONICET.

2 Departamento de Ciencias de la Administración, Universidad Nacional del Sur (DCA, UNS CONICET). E-mail: melisa.manzanal@uns.edu.ar

${ }^{3}$ DCA, UNS. E-mail: milanesi@uns.edu.ar

${ }^{4}$ Departamento de Economía, UNS; CEDETS - UPSO; CIC. E-mail: hvigier@uns.edu.ar
} 
heurísticas más observadas en los sujetos bajo análisis son la disponibilidad, la representatividad y la sobreconfianza. Esto ratifica la evidencia empírica resultante de estudios científicos previos, por lo que se aconseja considerar dichos aspectos conductuales en el estudio de las decisiones humanas y, particularmente, en las empresarias.

Palabras clave: heurísticas, empresarios pyme, panel de expertos.

\section{Abstract}

This article is part of a broader research project aimed at describing the decisions of SME entrepreneurs from Bahía Blanca, Argentina. In recent decades, mostly experimental studies have demonstrated the presence of heuristics and biases in human behaviour. Therefore, the objective of this article was to determine which heuristics are more likely used in the choices made by a particular universe of subjects, namely the SME entrepreneurs from the aforementioned city.

Thus, a panel of SME experts was selected. Two techniques were used to process the data obtained: probabilistic (absolute and cumulative frequencies) and non probabilistic (direct method with multiple experts). It was concluded that the most frequently observed heuristics in the subjects under analysis are availability, representativeness and overconfidence. This confirms the empirical evidence resulting from previous scientific research, so it is advisable to consider these behavioural aspects in the study of human decisions, particularly business-related decisions.

Keywords: heuristics, SME entrepreneurs, panel of experts.

JEL: D, 81D, 87M, 10. 


\section{Introducción}

El presente artículo presenta los resultados obtenidos en la primera fase de investigación concerniente a una tesis doctoral defendida en marzo de 2017. Esta última buscó caracterizar la toma de decisiones del empresario de la pequeña y mediana empresa (pyme). Concretamente, se estudió la presencia de las heurísticas y sesgos cognitivos en las elecciones de dichos sujetos.

La evidencia empírica resultante de diversos estudios científicos, mayormente experimentales, demuestra la existencia de heurísticas en la conducta de los individuos (Shefrin, 2010). Sin embargo, se supone que no todas las que constituyen el estado del arte en esta materia se encuentran presentes en todas las personas. De igual modo, dentro de aquellas que sí, se considera que no se manifiestan con la misma intensidad en cada individuo o grupo.

En consecuencia, este trabajo describe el diseño de un panel de expertos en pymes cuyos datos se procesan a partir de dos técnicas: probabilística y no probabilística o borrosa. Su objetivo es determinar qué heurísticas, de un compendio de ellas obtenido de la literatura, son más propensas a observarse en las decisiones de los empresarios de este tipo particular de empresas.

El artículo se estructura de la siguiente manera. En el siguiente apartado, se desarrolla un resumen de la revisión de la literatura necesaria para enmarcar el presente trabajo. A continuación, se describe la metodología abordaba en cuanto al diseño y procesamiento de los datos del panel de expertos. Luego se presentan los resultados obtenidos a partir de la aplicación de dos herramientas metodológicas previamente explicadas, y finalmente se exponen las consideraciones finales y la reseña bibliográfica.

\section{Revisión de la literatura}

Como se expone actualmente en la literatura, los sujetos no se comportan en el mundo real como sostienen las teorías neoclásicas, es decir, de modo totalmente racional y realizando elecciones óptimas. Sin embargo, la teoría económica de la utilidad esperada de von Neumann y Morgenstern (1944), que planteó un hombre completamente racional y omnipotente en el manejo de la 
información (Milanesi y El Alabi, 2011), mantuvo su hegemonía por años. Simon fue uno de los primeros en cuestionar el concepto de racionalidad ilimitada, proponiendo que este debía ser seriamente revisado. Sostuvo que lo más indicado es hablar de racionalidad limitada (Simon, 1955, 1978).

En consecuencia, surgieron las heurísticas y los sesgos. Tienen su raíz en diversos experimentos realizados por investigadores de la materia y en la teoría de las perspectivas de Kahneman y Tversky (1979), obra que desafió postulados clásicos, concretamente los axiomas de la teoría de la utilidad esperada. Se presentó como un modelo descriptivo, y alternativo a los existentes hasta entonces (Tversky y Kahneman, 1981). Por sus características y fundamentos generales, se considera que predice la mayoría de los comportamientos humanos (Viscusi, 1989).

Conceptualmente, las heurísticas son reglas de pulgar o rules of thumbs en la toma de decisiones, y los juicios son las valoraciones que realizan los individuos (Shefrin, 2010). Las personas forman juicios basándose en distintas heurísticas y estas sesgan sus elecciones de la conducta optimizadora y racional ilimitadamente plateada por los modelos neoclásicos (Shefrin, 2008, 2010).

No obstante, Kahneman (2011) 5 explicó que la existencia de sesgos de la intuición no implica dudar de la inteligencia humana. Por el contrario, la mayoría de los juicios y acciones de los agentes son apropiados. Actuar y tener la confianza en las propias creencias y preferencias suelen tener una base racional, aunque no siempre.

Shefrin (2010), en su libro Behavioralizing Finance ${ }^{6}$, realizó un resumen de las heurísticas existentes dentro del estado del arte. Dentro de las más estudiadas se pueden mencionar la representatividad, la sobreconfianza, la disponibili-

\footnotetext{
${ }^{5}$ En su obra Thinking fast and slow, el autor se centra en los sesgos de la intuición. Para ello, distingue entre dos grandes sistemas de pensamiento que definieron los psicólogos K. Stanovich y R. West y que coexisten en la mente humana: el sistema 1 y el sistema 2 . El primero es el intuitivo, rápido, que proviene de la emoción. Provee respuestas o elecciones automáticas. El sistema 2, en cambio, es aquel que surge de un proceso consciente, lento, racional y analítico (Kahneman, 2011; Stanovich y West, 2000).

${ }^{6}$ Las finanzas conductuales son aquellas que incorporan la psicología en el estudio de las finanzas, explicando las elecciones desde un enfoque conductual. Esto fue posible a partir de un cambio de paradigma en las finanzas tradicionales. Para mayor información, ver Barberis y Thaler (2003) y Shefrin $(2008,2010)$.
} 
dad, el status quo, el optimismo irreal, la extrapolación, la confirmación, el conservadurismo y el afecto (Bazerman, 2006; Gilovich, Griffin y Kahneman, 2002; Kahneman, 2003; Kahneman y Tversky, 1972; Kahneman, Slovic y Tversky, 1982; Shefrin, 2008, 2010; Tversky y Kahneman, 1973, 1974).

La representatividad es la tendencia de crear estereotipos para formar juicios. Permite que nuevos estímulos recibidos por un sujeto sean clasificados de manera eficiente, comparando los rasgos de ese nuevo estímulo con los ya existentes en un determinado prototipo o categoría (Kahneman, 2003). Al emplearse esta regla, el atributo objetivo de probabilidad es sustituido por el atributo de similitud más accesible (Kahneman y Tversky, 1972; Shefrin, 2010).

En concreto, sucede cuando las probabilidades son evaluadas por el grado en que " $A$ " es representativo o se asemeja a " $B$ ". Entonces, cuando " $A$ " es altamente representativo de " $\mathrm{B}$ ", la probabilidad que " $\mathrm{A}$ " se origine a partir de "B" es juzgada como alta (Tversky y Kahneman, 1974). En repetidos experimentos, Tversky y Kahneman han demostrado que el ser humano normalmente evalúa posibilidades mediante la representatividad, desestimando valores de probabilidades (objetivas) brindadas a priori.

La sobreconfianza es una heurística que se observa en personas que tienden a sobreestimar sus propios conocimientos y habilidades, creyendo que conocen más de lo que realmente saben de un tema o cuestión determinada (Shefrin, 2010). Una de las conclusiones a las que se arribó experimentalmente es que los individuos son sobreconfiados en cuanto a sus conocimientos cuando se trata de temas complejos. Entonces, tienden a establecer sus previsiones en intervalos que son demasiado estrechos (Shefrin, 2008). Así se demostró que esto implica subestimar el riesgo o la volatilidad (Odean, 1998). Por lo tanto, regirse por esta heurística puede provocar decisiones aventuradas y poco objetivas, basadas meramente en la intuición y los cálculos propios del empresario (Manzanal, Vigier, Briozzo, Milanesi y Martínez, 2015).

La disponibilidad surge a partir la facilidad en que ciertos elementos o recuerdos vienen rápidamente a la mente del sujeto para evaluar la frecuencia de una clase o la probabilidad de un evento determinado (Kahneman, 2003; Tversky y Kahneman, 1974). Es una heurística sumamente interesante que se observa en los individuos cuando tienden a evaluar la importancia relativa de 
ciertos asuntos según la propensión con que son traídos a la memoria. En consecuencia, los temas frecuentemente mencionados habitan en la mente, mientras otros escapan de la consciencia.

El status quo se manifiesta en los agentes que favorecen la inacción ante la acción, por la aversión al arrepentimiento. Esto implica permanecer en las posiciones que están y desestimar la nueva información que implique cambiar de rumbo (Milanesi y El Alabi, 2011). El optimismo irreal sucede cuando se sobreestima la posibilidad de un evento favorable y se subestima la de un evento desfavorable. La extrapolación induce a los sujetos a suponer estimaciones indeseadas sobre la base de la creencia de que cambios recientes continuarán en el futuro. El sesgo de confirmación incita a los individuos a sobreponderar la información que confirma sus posturas previas, y ponderar con menor peso la información que las rechaza.

En tanto, el conservadurismo es la heurística que se observa frente a la tendencia a no reaccionar ante nueva información (Milanesi y El Alabi, 2011; Shefrin, 2010). La heurística del afecto fue propuesta por Slovic, Finucane, Peters y MacGregor (2002). Se refiere a la emisión de juicios basándose en los sentimientos (Shefrin, 2010). Loewenstein, Weber, Hsee y Welch (2001) demostraron que la creencia que se tiene respecto de los riesgos, en muchas ocasiones, está fundada en las emociones personales. Kahneman, Wakker y Sarin (1997) plantearon que las decisiones evaluadas a partir del afecto no se ajustan a la lógica de las preferencias económicas.

Pese a que la incorporación formal de la psicología y, puntualmente, de las heurísticas en las teorías que explican la toma de decisiones data solo de unas décadas atrás, su aplicación en las empresas es un campo poco explorado. Sin embargo, previo a las teorías aquí mencionadas, Barnard (1938) probablemente haya sido uno de los primeros en reconocer como parte intrínseca del éxito de la labor de un gerente un elemento subjetivo: la intuición. En línea con la investigación de Simon (1955), sostuvo que el administrador cuenta con una vasta cantidad de conocimiento registrado en su memoria.

Por otra parte, Brownlie y Spender (1995), dedicados a estudiar la toma de decisiones en altos gerentes en el área de Marketing, apoyaron las ideas de Simon. Así mostraron que la mejor decisión en condiciones de alta incerti- 
dumbre se realiza sobre la base de juicios de los administradores o managers. Por consiguiente, atendiendo a que quien gerencia la pyme cumple mayormente el rol de propietario-administrador ${ }^{7}$ (Manzanal et al., 2015), se puede afirmar que ello genera un mayor nivel de subjetividad en la toma de decisiones empresarias (Briozzo y Vigier, 2009). Además, Busenitz y Barney (1997) probaron que los emprendedores son más propensos a recaer en sesgos y heurísticas al momento de tomar decisiones, en relación con los administradores de grandes empresas.

Analizando algunas heurísticas en particular, se puede mencionar a Alcalá Villarreal (2014), quien concluyó que los administradores financieros de la ciudad de Barranquilla realizan sus inversiones en mercados de renta fija, principalmente en valores nacionales. Esto permitió presumir la existencia del sesgo cognitivo de invertir en lo local, tal como en bonos de gobierno. Si bien se trata de instrumentos de inversión relativamente seguros, no siempre representan la decisión óptima desde la perspectiva racional clásica (maximización riqueza), debido a que no devengan el mayor rendimiento.

Otra investigación presentada por Lorenzana de la Varga y Lao Herrerías (2015) analizó la incidencia del género en las elecciones financieras de las empresas y la presencia de la heurística de la sobreconfianza, suponiendo que un mayor capital invertido sobre el total de activo es consecuencia de un exceso de confianza. No obtuvieron evidencia estadística suficiente para suponer que el porcentaje de capital invertido en el total activos se relaciona con si dichas decisiones empresarias son tomadas por mujeres $\mathrm{u}$ hombres.

En línea con el estudio de la sobreconfianza, Citci e Inci (2016) discutieron algunos hallazgos recientes en la literatura, demostrando que en ciertos casos el exceso de confianza de los administradores de una empresa podría ser una respuesta racional frente al entorno en el que operan, más que un rasgo personal. Ancarani, Di Mauro y D’Urso (2016) investigaron experimentalmente la

\footnotetext{
${ }^{7}$ En un estudio realizado en una muestra de 76 empresarios de pymes bahienses, se observó que el $92 \%$ de la muestra revestía la figura de empresario-administrador, siendo solo el $8 \%$ únicamente empresario, es decir, dueño o socio, sin participación en la administración (Manzanal et al., 2015). Esto genera lo que Briozzo y Vigier (2009) llamaron fenómeno "entrelazamiento empresa-propietario" (unificación entre la dirección y la propiedad).
} 
importancia del exceso de confianza en la gestión de la cadena de suministro. Los resultados arrojaron que la sobreconfianza puede ocasionar que los administradores de dicha especialidad sean menos cuidadosos en la gestión de los inventarios, incurriendo en mayores costos. Además, se probó la existencia del sesgo del optimismo en los gerentes de compras.

También es dable mencionar uno de los resultados preliminares y ya publicados de la mencionada tesis de la cual forma parte este artículo resumen. Según una muestra parcial de 76 empresarios pyme de la ciudad de Bahía Blanca, Argentina (Manzanal et al., 2015), los factores que mayormente orientan e influyen en las decisiones de inversión empresarias de estos sujetos son los rendimientos esperados (criterio de elección "racional u objetivo") y el afecto o apego por negocio (criterio de elección "heurístico o subjetivo").

De un análisis bivariado realizado con la misma muestra se concluyó que los empresarios más longevos, menos educados, que administran empresas más pequeñas y más antiguas toman decisiones de inversión basándose principalmente en criterios vinculados a heurísticas, como su apego al negocio, su intuición, su experiencia y las tendencias del sector al que pertenecen. En el análisis multivariado, las variables independientes: la edad del empresario y la antigüedad de la empresa, son las que incrementan la probabilidad de pertenecer al grupo de decisores que se rigen por heurísticas.

\section{Panel de expertos}

En este apartado se describe la metodología empleada para cumplir con el objetivo aquí propuesto que buscó determinar las heurísticas más observadas en las elecciones de los empresarios de pymes. Vale recordar que si bien este es el propósito principal del presente artículo, operó como insumo o primer objetivo para la mencionada tesis de la que forma parte.

La investigación doctoral pretendió testear experimentalmente la presencia de ciertos atajos mentales en las decisiones empresarias. Como tomar ad hoc algunas heurísticas y no otras para ser evaluadas en los experimentos podía ser objeto de críticas, se recurrió a un panel de expertos para definirlas, en 
virtud de su expertise y como respaldo de la elaboración de cuestionarios en una etapa siguiente de dicha tesis.

Hernández Sampieri et al. (2010) sostienen, a modo de aval metodológico, que el empleo de una muestra de expertos es lo más indicado en estudios donde resulta de gran utilidad obtener la opinión específica de especialistas. Permite elaborar hipótesis más precisas o ser la materia prima necesaria para la correcta confección de cuestionarios. Por lo tanto, se consideran muestras válidas cuando los objetivos de la investigación lo requieren.

\subsection{Diseño}

Todo panel se conforma de especialistas en un determinado tema, que para esta investigación son las pymes. A partir del panel, se hallan o confirman ideas y, por ende, su principal ventaja es la confiabilidad de la información obtenida, debido a que se basa en el conocimiento que justamente tienen los expertos. Otras de sus virtudes son el ahorro considerable de tiempo, su costo reducido o nulo, y la gran credibilidad de las conclusiones a las que se arriban (Manzanal et al., 2015).

Para crear un panel se precisa identificar y seleccionar cuidadosamente quienes lo integrarán (Hernández Sampieri et al., 2010). Esto se debe realizar de acuerdo con un determinado perfil previamente establecido, que asegure los requisitos de ser reconocidos y tener conocimiento en el área bajo análisis. Además, se sugiere garantizar la independencia de los especialistas respecto del programa o investigación para el cual son convocados como expertos (Manzanal et al., 2015).

Teniéndose en cuenta todas las particularidades precedentemente citadas, el panel objeto de este trabajo se integró por 31 especialistas en pymes de la ciudad de Bahía Blanca, Argentina. Concretamente, estuvo conformado por docentes e investigadores de Ciencias Contables, de Administración y Económicas de la Universidad Nacional del Sur (UNS) y la Universidad Provincial del Sudoeste (UPSO) y referentes de distintas instituciones y asociaciones locales que nuclean a este tipo particular de empresas. Todos ellos fueron cuidadosamente seleccionados por su conocimiento y contacto con empresarios 
bahienses de este tipo particular de empresas, ya sea desde el ámbito académico y/o profesional.

La herramienta de investigación empleada para contactarse con los expertos fue el cuestionario. Este se elaboró a través del formulario que ofrece Google y se envió por correo electrónico. Se estructuró con una sucinta introducción donde se explicaba el objetivo general de la investigación y el específico del cuestionario. Seguidamente, se definió el concepto de heurística, considerando que el grupo de especialistas escogidos lo era en pymes, pero no en aspectos conductuales. Luego se presentaron las nueve heurísticas (ver tabla 1) expuestas precedentemente en este marco teórico y una breve definición de ambas, para que los expertos pudieran realizar la actividad que se les solicitaba.

Tabla 1. Heurísticas empleadas en el Panel de Expertos

\begin{tabular}{c}
\hline HEURÍSTICAS \\
\hline Disponibilidad \\
Representatividad \\
Sobreconfianza \\
Optimismo Irreal \\
Extrapolación \\
Confirmación \\
Conservadurismo \\
Afecto \\
Status Quo \\
\hline
\end{tabular}

Fuente: elaboración propia.

La tarea encomendada a los integrantes del panel consistió en establecer, a su criterio, un orden de presencia de estos atajos mentales en las elecciones de los empresarios de pymes de Bahía Blanca. Metodológicamente, cada heurística bajo análisis se consideró una variable categórica cuya definición y recorrido se observa en la tabla 2. 
Tabla 2. Definición de la variable $X_{i}$

\begin{tabular}{|c|c|}
\hline VARIABLE $X_{i}$ & RECORRIDO $X_{i}$ \\
\hline $\begin{array}{c}\text { “Intensidad de la presencia de la } \\
\text { heurística } i \text { ". }\end{array}$ & $\begin{array}{c}\text { “Muy Presente”; “Presente”; ; Poco Presen- } \\
\text { te"; "No Presente"; “NS/NC" (no sabe, no }^{\text {contesta). }}\end{array}$ \\
\hline
\end{tabular}

Fuente: elaboración propia.

Los especialistas solo podían escoger uno de estos cinco elementos del recorrido. Los primeros cuatro indicaron nivel de presencia de cada heurística en las decisiones de los mencionados sujetos. El valor de la variable "NS/NC", en cambio, se incorporó para los casos en los que no tenían conocimiento alguno respecto de si una determinada heurística se encuentra presente, o no, en la conducta del empresario. Así, se evitó obligar al experto a que opte por algún nivel de presencia de cierta heurística, cuando en realidad lo desconocía.

\subsection{Procesamiento de los datos}

Para procesar los datos resultantes del panel se emplearon dos técnicas. Una fue de base probabilística (frecuencias acumuladas relativas porcentuales) y la otra no probabilística o borrosa (fuzzy: funciones de pertenencia construidas a partir del método directo con múltiples expertos). Si bien son disímiles entre sí, ambas permiten cuantificar la incertidumbre. A continuación, se explica el origen y cálculo de cada una.

\subsubsection{Base probabilística}

La falta de certeza se describe de muchas maneras, pero Tversky y Kahneman (1974) plantearon que aquella que brinda una mejor descripción de cualquier evento incierto es la expresión numérica de la probabilidad. Conceptualmente, es una medida numérica de la posibilidad de que ocurra un evento, entendiéndose por este último un resultado experimental. 
De este modo, las probabilidades se usan como medidas de falta de certeza asociada a eventos o estados de la naturaleza. Los valores de probabilidad están comprendidos entre cero y uno, y su recorrido representa grados posibles de ocurrencia del mismo (Anderson et al., 2011). Otra forma de definir la probabilidad es como una medida del nivel de incertidumbre de cada suceso o evento aleatorio (Martín-Pliego y Ruiz-Maya, 2006).

Existen tres grandes enfoques dentro del estado del arte para asignar probabilidades (Lind, Marchal y Wathen, 2008). El primero es el de la probabilidad clásica, basado en la suposición de que los resultados de un experimento son igualmente viables, es decir, equiprobables. Así, este enfoque define la probabilidad para un evento como el número de casos o resultados favorables dividido el número total de casos o resultados posibles.

El segundo enfoque es el de la probabilidad empírica. Trabaja con las frecuencias relativas. Esto implica que la probabilidad de ocurrencia de un evento se determina al observar el número de veces que este sucedió en el pasado (Landro, 2010).Tanto la probabilidad empírica como la clásica se consideran objetivas.

Sin embargo, para los casos en que existe poca o ninguna experiencia o información previa sobre la cual basar la probabilidad, lo indicado es emplear el tercer y último enfoque: el subjetivo (Savage, 1954). Es aquel basado en las creencias que tiene un individuo respecto de un estado de la naturaleza. Se obtiene con la evaluación que realiza el sujeto de las opiniones y de la información disponibles, para luego asignar una probabilidad de ocurrencia a un determinado suceso (Lind et al., 2008).

En conclusión, más allá del enfoque que se emplee, la probabilidad constituye un elemento importante, debido a que proporciona una forma de medir, expresar y analizar la incertidumbre asociada con eventos futuros.

A fin de determinar el nivel de presencia de cada heurística en las decisiones empresarias de pymes bahienses, se utilizó como herramienta de base probabilística el enfoque empírico, en concreto, las frecuencias absolutas rela- 
tivas y acumuladas porcentuales ${ }^{8}$. La tasa de corte definida ad hoc para determinar si cada sesgo está presente o no en las elecciones de estos sujetos fue del $75 \%$ de la frecuencia acumulada porcentual del valor "presente". Esto tiene su fundamento en que al utilizarse dicha frecuencia, el valor de la misma para la categoría "presente" incluye o acumula la frecuencia relativa (frecuencia observada sobre el total de expertos) del valor "muy presente" y "presente" donde, en definitiva, ambas indican presencia.

\subsubsection{Base no probabilística o borrosa}

Otra forma de cuantificar la falta de certeza es la no probabilística o borrosa. Tiene su raíz en la teoría de la posibilidad propuesta por Lotfi Zadeh, con el fin de manipular formalmente la imprecisión y la vaguedad del razonamiento humano (Zadeh, 1978). Puesto que la presencia de incertidumbre se origina a partir de la existencia de información incompleta, por ende ambigua; dicha impresión es posible explicarla mediante números borrosos (Dubois, 2010; Gil-Aluja, Terceño Gómez, Ferrer Comalat, Merigó-Lindahl y Linares Mustaros, 2015; Kuchta, 2000; Terceño, Barberá, Vigier y Laumann, 2011).

Los números borrosos son una expresión de posibilidad o creencia acerca de algo. Concretamente, un número borroso se constituye a partir de una familia de intervalos que representa la estimación de una magnitud. La aritmética borrosa (fuzzy arithmetic) permite efectuar cálculos con cantidades numéricas que son conocidas de forma imprecisa. Dicha impresión se expresa entre 0 y 1 , definiéndose con una distribución unimodal que llega a la unidad para uno o varios valores (Fornero, 2012).

\footnotetext{
${ }^{8}$ La frecuencia absoluta para una categoría, valor o clase de una determinada variable indica el número de observaciones en la muestra (o población), con ese valor o rango. Se expresa con la variable $f_{i}$, donde la sumatoria de los $f_{i}$ es el total de la muestra $(n)$ (o población $(N)$, según el caso): $\sum_{i=1}^{n} f_{i}=n$. Luego la frecuencia relativa $f_{r}$ se calcula como $f_{r}=\frac{f_{i}}{n}$. La frecuencia acumulada $\left(F_{a}\right)$, en cambio, es la suma de las frecuencias absolutas de los valores (o categorías) inferiores o iguales al valor de $x_{i}$ considerado. La frecuencia acumulada porcentual se calcula como: $F_{a} \%=\sum \frac{f_{i}}{n} \times 100$.
} 
Las funciones de pertenencia de un número borroso se interpretan como una distribución de posibilidad de pertenencia a un conjunto dado. Se pueden determinar aplicando criterios individuales (subjetivos y objetivos), colectivos, procedimientos analíticos y procedimientos experimentales (Trillas, Alsina y Terricabras, 1995). A su vez, para la construcción de funciones de pertenencia existen métodos directos, métodos indirectos y métodos que utilizan la frecuencia relativa.

En los métodos directos, el experto brinda respuestas a preguntas de varios aspectos que explícitamente pertenecen a la función de pertenencia a construir. En los métodos indirectos, en cambio, se requiere que los expertos respondan preguntas simples y menos dependientes de las diferentes bases de juicio subjetivo, las cuales pertenecen implícitamente a la función de pertenencia construida. De esta forma, las respuestas son procesadas posteriormente de acuerdo con diferentes supuestos realizados. En tanto, los métodos directos e indirectos se clasifican en métodos de un experto y métodos de múltiples expertos.

El método que se empleó en este estudio es directo con varios expertos. Para comprenderlo, es necesario explicar cómo opera el método directo con un experto. En este último, el sujeto asigna a cada elemento dado $x \& X$ un grado de pertenencia $A(x)$ que, según su opinión, captura mejor el significado del término lingüístico representado por el subconjunto borroso $A$. Esto puede ser realizado definiendo la función de pertenencia a partir de una fórmula matemática, o bien ejemplificando esta para algunos elementos seleccionados de $\mathrm{X}$.

Cuando un método directo es extendido a varios expertos, como en este trabajo, las opiniones individuales deben ser agregadas. Una de las formas más usuales se basa en funciones de pertenencia de interpretación probabilística. Supone que $n$ expertos son consultados sobre algunos elementos $x \in X$ para valuar la proposición: " $x$ pertenece a $A$ ", como si fuera falso o verdadero, y donde $A$ es un subconjunto borroso sobre $X$, que representa un término lingüístico asociado con una variable lingüística dada.

Para un elemento particular $x \in X$, se denota como $a_{i}(x)$ la respuesta del experto $i$, con $i=1,2, \ldots n$, siendo $a_{i}(x)=1$ cuando la proposición se valúa por el ex- 
perto $i$ como verdadera y $a_{i}(x)=0$ cuando la considera falsa. En la siguiente ecuación se visualiza la función de pertenencia construida.

$$
A(x)=\sum_{i=1}^{n} \frac{a_{i}(x)}{n}
$$

Comúnmente se generaliza esta interpretación, permitiendo asignar ponderaciones o pesos: $c_{i}$, a la opinión de cada experto. Por lo tanto, $A(x)$ queda expresada como:

$$
A(x)=\sum_{i=1}^{n} c_{i} \times a_{i}(x) / n
$$

donde:

$$
\sum_{i=1}^{n} c_{i}=1
$$

Se debe tener en cuenta que en ciertas situaciones se considera una desventaja que los métodos directos requieran que el experto proporcione respuestas precisas para capturar los juicios subjetivos. Esto se debe a que las respuestas suelen ser algo arbitrarias, y esto puede ser criticado para ciertos conceptos complejos. Por ello la literatura propuso los métodos indirectos, que sustituyen la estimación directa del grado de pertenencia por una tarea simple que es determinada por el investigador y le es encomendada al experto, y de cuya respuesta puede deducirse la función de pertenencia (Vigier, 2001).

No obstante, lo dicho precedentemente no representa un problema o desventaja dentro de este estudio. Si bien el elemento subjetivo está presente, como en toda situación susceptible de ser analizada a partir de la lógica borrosa, no interfiere en las categorías previamente definidas para darle un grado de pertenencia a cada heurística $(X)$ en el conjunto "empresarios de pymes".

De acuerdo con el proceso de valuación directa de cada experto consultado, se procedió a aplicar el método directo con varios expertos. A partir del formulario utilizado para captar las opiniones, se identificaron cuatro etiquetas lingüísticas que representan el nivel de presencia de cada heurística en el proceso de toma de decisiones de los sujetos bajo análisis, a juicio de los ex- 
pertos seleccionados. Los valores que se asignaron para cada una de dichas etiquetas son los que se presentan en la tabla 3:

Tabla 3. Pesos de cada etiqueta (o nivel de presencia) lingüística

\begin{tabular}{cc}
\hline Etiqueta & Valor \\
\hline Muy Presente & 1 \\
Presente & 0,66 \\
Poco Presente & 0,33 \\
No Presente & 0 \\
\hline
\end{tabular}

Fuente: elaboración propia.

Obtenida la opinión de cada experto, se realizó la agregación por heurística, suponiendo que la importancia de la respuesta de cada uno era la misma. Es decir, a partir de la etiqueta elegida para cada heurística, se determinó el nivel de pertenencia o presencia de ellas en el conjunto de los empresarios pyme. Las heurísticas seleccionadas como presentes en las decisiones de empresarios pyme fueron aquellas cuya valuación agregada fue mayor o igual a 0,66 (tasa de corte definida ad hoc para este método); es decir, del valor de la etiqueta lingüística "presente".

Finalmente, las heurísticas con las que se concluye esta investigación serían las coincidentes en cada método, es decir, el probabilístico y el borroso. Emplear estas dos técnicas permitiría contrastar y validar los resultados obtenidos en cada una, considerando que el producto de este artículo sería el insumo para una etapa siguiente ${ }^{9}$ de la tesis doctoral de la cual forma parte.

${ }^{9}$ Dicha etapa se refiere a los casos hipotéticos para probar la presencia de heurísticas en un pseudoexperimento diseñado para empresarios pyme de Bahía Blanca. 


\section{Resultados del panel}

Este apartado expone las heurísticas más propensas a ser observadas en las decisiones de los empresarios pyme de Bahía Blanca. Se obtuvieron 18 respuestas del cuestionario enviado a 31 expertos. A continuación, se presentan los resultados por cada una de las técnicas anteriormente explicadas.

\subsection{Análisis probabilístico: frecuencias acumuladas}

Los datos relevados del cuestionario entregado al panel de expertos vía Google fueron procesados con el software SPSS. Por cada heurística de las nueve analizadas se calcularon y analizaron las tablas de frecuencias absolutas, relativas y acumuladas. A modo ilustrativo, se exhiben las tablas de frecuencias de dos heurísticas (ver tabla 4 y tabla 5).

Por un lado, como se explicó en el aparatado anterior y se observa en las tablas expuestas, la categoría "No Sabe/No Contesta" ("NS/NC") fue excluida del recorrido de cada variable (heurística) al momento de procesar los datos obtenidos, por no indicar nivel de presencia alguno. En consecuencia, en los casos donde no se eligió dicho valor de variable, el total de respuestas ( $n$ ) fue exactamente de 18, pero en aquellos en que sí, el total indefectiblemente fue menor, por excluir las observaciones para la mencionada categoría.

Tabla 4. Cuadro de frecuencias de la heurística de la extrapolación ${ }^{\mathbf{1 0}}$

\begin{tabular}{lccc}
\hline $\begin{array}{c}\text { Recorrido de la } \\
\text { variable }\end{array}$ & $\begin{array}{c}\text { Frecuencia } \\
\text { absoluta }\end{array}$ & $\begin{array}{c}\text { Frec. abs. relativa } \\
\mathbf{( \% )}\end{array}$ & $\begin{array}{c}\text { Frec. acumulada } \\
\mathbf{( \% )}\end{array}$ \\
\hline Muy presente & 1 & 6,7 & 6,7 \\
Presente & 7 & 46,7 & 53,3 \\
Poco Presente & 6 & 40,0 & 93,3 \\
No Presente & 1 & 6,7 & 100,0 \\
Total & 15 & 100,0 & \\
\hline
\end{tabular}

Fuente: elaboración propia.

${ }^{10}$ Como se visualiza en la tabla, el total de respuestas fue menor a 18 , concretamente: $\sum_{i=1}^{n} f_{i}=$ $n=15$. Esto demuestra que existieron tres observaciones descartadas del procesamiento y análisis de los datos, por encontrarse en la categoría "NS/NC". 
Tabla 5. Cuadro de frecuencias de la heurística de la confirmación ${ }^{11}$

\begin{tabular}{lccc}
\hline $\begin{array}{c}\text { Recorrido de la } \\
\text { variable }\end{array}$ & $\begin{array}{c}\text { Frecuencia } \\
\text { absoluta }\end{array}$ & $\begin{array}{c}\text { Frec. abs. relativa } \\
\mathbf{( \% )}\end{array}$ & $\begin{array}{c}\text { Frec. acumulada } \\
\mathbf{( \% )}\end{array}$ \\
\hline Muy presente & 7 & 38,9 & 38,9 \\
Presente & 6 & 33,3 & 72,2 \\
Poco Presente & 5 & 27,8 & 100,0 \\
No Presente & 0 & 0 & 100,0 \\
Total & 18 & 100,0 & \\
\hline
\end{tabular}

Fuente: elaboración propia.

Por otro lado, en virtud a la tasa de corte definida para el análisis probabilístico (75\% de la frecuencia acumulada relativa del valor "presente"), las heurísticas consideradas con mayor presencia en la toma de decisiones de empresarios pyme (según los expertos) serían aquellas cuya frecuencia acumulada relativa porcentual fuera mayor o igual a dicho valor.

Por lo tanto, se concluye que las heurísticas más propensas a ser vistas en el proceso decisorio del empresario de pymes bahienses a juicio de los expertos seleccionados y según la técnica probabilística son la disponibilidad, la representatividad y la sobreconfianza (ver tabla 6).

Tabla 6. Resumen de frecuencia acumulada (\%) del valor "presente" por heurística

\begin{tabular}{cc}
\hline Heurística & Frecuencia acumulada relativa (\%) \\
\hline Disponibilidad & $\mathbf{8 8 , 2}$ \\
Representatividad & $\mathbf{8 8 , 2}$ \\
Sobreconfianza & $\mathbf{7 7 , 8}$ \\
Confirmación & $\mathbf{7 2 , 2}$ \\
Optimismo irreal & 61,1 \\
Extrapolación & 53,3 \\
Conservadurismo & 44,4 \\
Afecto & 44,4 \\
Status quo & 38,9 \\
\hline
\end{tabular}

Fuente: elaboración propia.

${ }^{11}$ Para el procesamiento de esta heurística, el total de observaciones resultó ser de 18 (exactamente igual al $n=18$ expertos). Además, la categoría "no presente" no obtuvo ninguna observación; es decir, ningún experto consideró que esta heurística no está presente en las elecciones de los empresarios de pymes bahienses. 


\subsection{Análisis borroso: método directo con múltiples expertos}

La segunda herramienta con la que se procesaron los datos del panel proviene de la matemática borrosa (fuzzy logic), y se refiere a la construcción de funciones de pertenencia. En este caso, se aplicó el método directo con múltiples expertos.

Consecuentemente, se procedió a realizar el debido cálculo. De la ecuación 3 (descripta en el apartado 3.2.2): $A(x)=\sum_{i=1}^{n} c_{i} \times a_{i}(x) / n$, donde $c_{i}$ representa la ponderación correspondiente a cada nivel de presencia (ver tabla 3); $a_{i}(x)$ la respuesta de cada experto $i$, y $n$ la cantidad de expertos que la calificaron con algún nivel de presencia a cada atajo mental; se efectuó la valuación agregada (múltiples expertos) por cada heurística ${ }^{12}$ (ver tabla 7).

Tabla 7. Valuación agregada por heurística

\begin{tabular}{cc}
\hline Heurística & Valuación Agregada \\
\hline Disponibilidad & $\mathbf{0 , 7 4 1 2}$ \\
Confirmación & $\mathbf{0 , 7 0 0 6}$ \\
Representatividad & $\mathbf{0 , 6 8 1 8}$ \\
Sobreconfianza & $\mathbf{0 , 6 6 2 8}$ \\
Optimismo Irreal & 0,5322 \\
Extrapolación & 0,5067 \\
Afecto & 0,4967 \\
Conservadurismo & 0,4789 \\
Status Quo & 0,3672 \\
\hline
\end{tabular}

Fuente: elaboración propia.

De esta agregación de respuestas, a partir de la opinión de cada experto y considerando la tasa de corte definida para el análisis borroso, las heurísticas que encabezan las elecciones de los empresarios resultaron ser la disponibilidad, la confirmación, la representatividad y la sobreconfianza.

\footnotetext{
12 Es decir, la suma ponderada del peso otorgado a cada categoría o valor de la variable ("muy presente", "presente", "poco presente" y "no presente") por la cantidad de observaciones o expertos que eligieron esa categoría o valor; dividido el total de expertos $(n)$ que calificaron a esa heurística (cualquiera sea la categoría escogida).
} 


\subsection{Resultado final del panel}

Finalmente, tomando los resultados obtenidos del panel según el empleo de las técnicas de procesamiento de datos probabilística y no probabilística, las heurísticas coincidentes fueron: la disponibilidad, la representatividad y la sobreconfianza.

\section{Consideraciones finales}

Tal como se esbozó precedentemente, este artículo forma parte de una tesis doctoral cuyo fin fue caracterizar la toma de decisiones de un universo particular de sujetos: los empresarios de pymes de la ciudad de Bahía Blanca. En las últimas décadas muchos autores se han dedicado a estudiar y probar la existencia de diversas heurísticas y sesgos en el accionar de los individuos. Este nuevo cuerpo de conocimiento complementa los modelos de elección hasta entonces estudiados, presentando supuestos más reales para describir y explicar el comportamiento humano. Por consiguiente, el objetivo del presente trabajo fue determinar qué heurísticas son más propensas a observarse en las decisiones de empresarios pyme de dicha ciudad.

Para ello, se recurrió a una muestra de expertos constituida por docentes e investigadores de dos universidades nacionales y referentes de instituciones que agrupan o representan a las pymes, todos de la citada ciudad. Como el diseño del panel no solo permitía satisfacer el objetivo aquí planteado, sino también ser insumo para una etapa siguiente de la mencionada investigación doctoral, se decidió contrastar y validar los resultados empleando dos técnicas de procesamiento de los datos. Una de ellas fue probabilística, a partir del empleo de tablas de frecuencias absolutas y acumuladas, y otra no probabilística o borrosa, aplicando el método directo con múltiples expertos.

Procesada la información obtenida con cada herramienta, se concluyó que las heurísticas más observadas en los sujetos bajo análisis, según los expertos seleccionados, son la disponibilidad, la representatividad y la sobreconfianza. Esto ratifica la evidencia empírica resultante de estudios científicos previos que demuestran la existencia de un compendio de heurísticas en la conducta 
de los individuos, que en este caso fueron los empresarios de un tipo particular de empresas.

Finalmente, si bien los resultados obtenidos no constituyen postulados universales respecto de la conducta de estos sujetos, queda en evidencia la importancia del estudio y caracterización del componente subjetivo en los modelos de toma de decisiones en condiciones de incertidumbre, ambigüedad o información incompleta. Por lo tanto, se aconseja considerar aspectos conductuales, como lo son las heurísticas y los sesgos, en el estudio de las decisiones humanas y en concreto de las empresarias.

\section{Referencias}

Alcalá Villarreal, J. L. (2014). Análisis de los factores cognitivos que inciden en la toma de decisiones de inversión de los administradores financieros de la ciudad de Barranquilla. Revista Universidad Externado de Colombia, (24). Recuperado el 10 de noviembre de 2015 de http:/ / revistas.uexternado.edu.co/ index.php/sotavento/article/view/4054.

Ancarani, A., Di Mauro, C. \& D'Urso D. (2016). Measuring overconfidence in inventory management decisions. Journal of Purchasing $\mathcal{E}$ Supply Management, (22), 171-180.

Anderson, D., Sweeney, D., Williams, T., Camm, J. \& Martin, K. (2011). Métodos Cuantitativos para los negocios. 11. ${ }^{a}$ edición. DF, México: Cengage Learning Editores.

Barnard, C. I. (1938). The Functions of the Executive. Cambridge, Inglaterra: Harvard University Press.

Bazerman, M. H. (2006). Judgment in Managerial Decision Making. 6. a edición. Nueva York, USA: John Wiley \& Sons.

Briozzo, A. \& Vigier H. (2009). La estructura de capital de las pequeñas y medianas empresas argentinas. Estudios Económicos, 26(53). Recuperado el 20 de mayo de 2016 de http://bibliotecadigital.uns.edu.ar/scielo.php?script=sci_ arttext\&pid=S0425-368X2009002200001.

Brownlie D., \& Spender J. C. (1995). Managerial judgment in strategic marketing: some preliminary thoughts. Management Decision, 33(6), 39-50. 
Busenitz, L. W. \& Barney, J. B. (1997). Organizations: Biases and Heuristics in Strategic Decision-Making. Journal of Business Venturing, (12), 9-30.

Citci S. H. \& Inci, E. (2016). Career concerns and Bayesian overconfidence of Managers. International Journal of Industrial Organization, (46), 137-159.

Dubois, D. (2010). Representation, propagation and decision issues in risk analysis under incomplete probabilistic information. Risk Analysis, 30(3), 361-368.

Fornero, R. A. (2012). Valor de Proyectos de Inversión con estimaciones probabilísticas y borrosas. XXXII Jornadas Nacionales en Administración Financiera, Valle Hermoso, Argentina.

Gil-Aluja, J., Terceño Gómez, A., Ferrer Comalat, J. C., Merigó-Lindahl, J. M. \& Salvador Linares Mustaros, S. (2015). Scientific Methods for the Treatment of Uncertainty in Social Sciences. Nueva York, USA: Springer.

Gilovich, T., Griffin, D. \& Kahneman, D. (2002). Heuristics and Biases: The Psychology of Intuitive Judgement. Nueva York: Cambridge University Press.

Hernández Sampieri, R., Fernández Collado, C. \& Baptista Lucio, M. P. (2010). Metodología de la Investigación. 5. edición. DF, México: Mc Graw Hill.

Kahneman, D. (2003). Mapas de racionalidad limitada: Psicología para una Economía Conductual. Revista Asturiana de Economía, (28), 181-225.

Kahneman, D. (2011). Thinking, fast and slow. Nueva York, USA: Farrar, Straus and Giroux.

Kahneman, D. \& Tversky, A. (1972). Subjective Probability: a Judgment of Representativeness. Cognitive Psychology, (3), 430-454.

Kahneman, D. \& Tversky, A. (1979). Prospect Theory: An Analysis of Decision under Risk. Econométrica, 47(2), 263-291.

Kahneman, D., Wakker, P. P. \& Sarin, R. (1997). Back to Bentham? Explorations of Experienced Utility. Quarterly Journal of Economics, 112(2), 375-405

Kuchta, D. (2000): Fuzzy Capital Budgeting. Fuzzy Sets and Systems, 111(3), 367-385.

Lind, D. A., Marchal, W. G. \& Wathen, S. A. (2008). Estadística aplicada a los Negocios y la Economía. 13. ${ }^{a}$ edición. DF, México: Mc Graw Hill.

Loewenstein, G., Weber, E. U., Hsee, C. K. \& Welch N. (2001). Risk as Feeling. Psychological Bulletin, 127(2), 267-286. 
Lorenzana de la Varga \& Lao Herrerías (2015). Finanzas Conductuales: La presencia de mujeres en los consejos de administración y su influencia sobre algunas decisiones financieras. Comparativa IBEX35 y DAX30. XVIII Congress of the International Association for fuzzy - set Management and Economy (SIGEF), Girona, España.

Manzanal, M. N., Milanesi, G. S., Vigier, H. P. \& Toscana, L. (2015). Análisis del impacto de heurísticas en la toma de decisiones en condiciones de incertidumbre en las PyMEs. Revista EPIO, (37), 47-64.

Manzanal, M. N., Pesce, G., Milanesi, G. S., Vigier, H. P. \& Terceño, A. (2015). Razón versus Intuición: ¿Cómo deciden los empresarios de PyMEs? XX Reunión Anual de Red PyMES del Mercosur, Bahía Blanca, Argentina.

Manzanal, M. N., Vigier, H. P., Briozzo, A., Milanesi, G. S. \& Martínez, L. (2015). Construcción de funciones de pertenencia a partir del uso de la teoría de expertos. Medición de la presencia de heurísticas en la toma de decisiones. XVIII Congress of the International Association for fuzzy - set Management and Economy (SIGEF), Girona, España.

Martín-Pliego, F. J. \& Ruiz-Maya, L. R. (2006). Fundamentos de Probabilidad. 2. edición. Madrid, España: Thomson Editores y Paraninfo.

Milanesi, G. \& El Alabi, E. (2011). Toma de decisiones en condiciones de incertidumbre y la heurística de la Representatividad. X Congreso Internacional de Administración "La Gestión en Transformación" - Los retos de una nueva época. Buenos Aires, Argentina.

Odean, T. (1998). Volume, Volatility, Price, and Profit When All Traders Are Above Average. The Journal of Finance, 53(6), 1887-1934.

Savage L. J. (1954). The foundations of Statistics. Nueva York: John Wiley \& Sons.

Shefrin, H. (2008). A behavioral approach to asset pricing, 2. a edición. Nueva York: Academic Press Advanced Finance Series.

Shefrin, H. (2010). Behavioralizing Finance. Foundations and Trends in Finance, 4(1-2), 1-184.

Simon H. A. (1955). A Behavioral Model of Rational Choice. The Quarterly Journal of Economic, 69(1), 99-118.

Simon H. A. (1978). Rationality as Process and Product of Thought. The American Economic Review, 68(2), 1-16. 
Slovic, P., Finucane, M., Peters, E. \& MacGregor, D. G. (2002). Rational actors or rational fools: Implications of the affect heuristic for behavioral economics. The Journal of Socio-Economics, 31(4), 329-342.

Terceño, A., Barberà, G., Vigier, H. P. \& Laumann, Y. (2011). Coeficiente beta en sectores del mercado español: Regresión borrosa vs regresión ordinaria. Cuadernos del CIMBAGE, (13), 79-105.

Trillas, E., Alsina, C. \& Terrricabras, J. M. (1995). Introducción a la Lógica Borrosa. Barcelona, España: Editorial Ariel.

Tversky, A., \& Kahneman, D. (1973). Availability: A heuristic for judging frequency and probability. Cognitive Psychology, 5(2), 207-232.

Tversky A., \& Kahneman D. (1974). Judgment under Uncertainty: Heuristics and Biases. Science, New Series, 185(4157), 1124-1131.

Tversky, A., \& Kahneman, D. (1981). The Framing of Decisions and the Psychology of Choice, Science, (211), 453-458.

Vigier, H. (2001). Modelos de Diagnóstico de Empresas. Aplicación de la Resolución de Ecuaciones en Relaciones Difusas. Tesis Doctoral. Facultad de Ciencias Económicas y Empresariales. Universidat Rovira i Virgili. Reus, España.

Viscusi, W. K. (1989). Prospective reference theory: Toward an explanation of the paradoxes. Journal of Risk and Uncertainty, 2(3), 235-263.

Von Neumann, J. \& Morgenstern, O. (1944). Theory of Games and Economic Behavior. New Jersey, USA: Princeton University Press.

Zadeh L. A. (1978). Fuzzy sets as a basis for a theory of possibility. Fuzzy Sets and Systems, 1(1), 3-28.

(C) 2016 por los autores; licencia otorgada a la revista Escritos Contables y de Administración. Este artículo es de acceso abierto y distribuido bajo los términos y condiciones de una licencia Atribución-No Comercial 4.0 Internacional (CC BY-NC 4.0) de Creative Commons. Para ver una copia de esta licencia, visite https://creativecommons.org/licenses/by-nc/4.0/ 